Relations industrielles

Industrial Relations

\title{
La participation des travailleurs aux décisions dans les entreprises, Bureau international du travail, Genève, dans Relations professionnelles, no 33, 1969, 192 pp.
}

\section{Jean-Pierre Beaulieu}

Volume 26, numéro 1, 1971

URI : https://id.erudit.org/iderudit/028211ar

DOI : https://doi.org/10.7202/028211ar

Aller au sommaire du numéro

Éditeur(s)

Département des relations industrielles de l'Université Laval

ISSN

0034-379X (imprimé)

1703-8138 (numérique)

Découvrir la revue

Citer ce compte rendu

Beaulieu, J.-P. (1971). Compte rendu de [La participation des travailleurs aux décisions dans les entreprises, Bureau international du travail, Genève, dans Relations professionnelles, no 33, 1969, 192 pp.] Relations industrielles / Industrial Relations, 26(1), 256-257. https://doi.org/10.7202/028211ar

Tous droits réservés @ Département des relations industrielles de l'Université Laval, 1971
Ce document est protégé par la loi sur le droit d'auteur. L'utilisation des services d’Érudit (y compris la reproduction) est assujettie à sa politique d'utilisation que vous pouvez consulter en ligne.

https://apropos.erudit.org/fr/usagers/politique-dutilisation/ 
Nous croyons que le volume de Maier s'avère encore un guide extrêmement utile dans la conduite de type d'entretien.

Après avoir décrit les buts de l'entretien d'appréciation, Maier procède à l'aide de cas concrets à la description et à l'analyse critique de chacun des trois modes d'entretien qu'il a retenus:

- la première méthode (dénommée ici « juge et vends 》) consiste à communiquer l'appréciation et à persuader le subordonné de s'améliorer, le supérieur se cantonnant dans son rôle de juge;

- par la deuxième méthode («juge et fais parler »), le supérieur communique l'appréciation et, au cours d'un entretien de type non directif, essaie de faire tomber les défenses de son interlocuteur ;

- la troisième méthode (dite « résolutive $\gg)$ consiste à ne communiquer au départ aucune appréciation. Supérieur et subordonné, au cours d'un dialogue véritable, s'efforcent de découvrir en commun ce qui ne cadre pas avec les objectifs visés et de rechercher ensemble les solutions. Le supérieur n'a plus le rôle de juge, mais celui d'aide. Son autorité ne repose plus sur son savoir et sa position dans la hiérachie, mais sur le fait qu'il assume une fonction propre à répondre aux besoins du subordonné quant à la réalisation des buts visés.

Les jeux de rôles et les analyses qui suivent permettent de saisir les inconvénients et les avantages de chaque mode d'entretien. Le premier mode accentue la dépendance du subordonné à l'endroit du supérieur sans lui donner l'occasion de faire valoir son propre point de vue. Le deuxième donne cette possibilité au subordonné d'expliquer les difficultés qu'il rencontre dans l'accomplissement de sa tâche. C'est aussi l'occasion de rationaliser son comportement tout en laissant croire à son supérieur que des améliorations vont s'en suivre. L'approche résolutive présente beaucoup de difficultés dans la pratique, puisqu'elle suppose chez les subordonnés la capacité de réagir positivement à l'endroit des personnes en position d'autorité. Cette dernière approche s'accommode bien avec les théories récentes en administration des entreprises qui accordent une place importante à la participation dans la prise de décision et qui, par conséquent, viennent modifier les systèmes courants de rotation du personnel.

\section{Laurent BELANGER}

La participation des travailleurs aux décisions dans les entreprises, Bureau international du travail, Genève, dans Relations professionnelles, no 33, 1969, 192 pp.

Le présent numéro de la série Relations Professionnelles nous offre le rapport d'une réunion technique sur les droits des représentants syndicaux et la participation des travailleurs aux décisions dans les entreprises, réunion tenue à Genève en novembre 196\%. A la suite de cette réunion, ce rapport fut soumis au Conseil d'administration qui autorisa le Directeur général du BIT « à donner une large diffusion au rapport adopté par la Réunion ainsi qu'à la documentation établie par celle-ci $\gg$. Il convient de mentionner que cette réunion technique réunissait dix-huit experts des questions de relations du travail, experts provenant de quinze pays européens, asiatiques et américains. Trois grands points sont traités dans ce document.

Le chapitre premier aborde dans son ensemble la question de la participation des travailleurs aux décisions dans les entreprises : concept, objectifs, méthodes, domaines. Ce chapitre nous est apparu particulièrement dense.

Le second chapitre traite plus spécifiquement des mécanismes de participation des travailleurs aux décisions, de leur nature et leur portée. Il s'agit d'un exposé des principaux types de mécanismes de participation en usage dans différents pays et diverses parties du monde en indiquant leurs principales caractéristiques, à commencer par la nature des pouvoirs accordés aux travailleurs ou à leurs représentants. C'est un tour d'horizon des plus intéressant.

Le troisième et dernier chapitre, excepté le résumé et les remarques finales, porte sur les divers domaines de l'entreprise pouvant faire l'objet de la participation des travailleurs. Les auteurs s'efforcent de donner «une idée d'ensemble des activités de l'entreprise 
auxquelles s'appliquent les différents mécanismes de participation analysés au chapitre II. II y aurait les décisions reliées aux politiques générales et celles reliées à des domaines déterminés de l'activité de l'entreprise : aspects économiques, emploi et politique de personnel, rémunération et conditions de travail, bien-être et plan socio-culturel.

Résultat de discussions d'experts dans ce domaine, basé sur une documentation des plus riches, ce rapport est d'un intérêt considérable pour tous ceux qui s'intéressent au problème de la participation des travailleurs aux décisions de lentreprise et à ses solutions.

\section{Jean-Pierre BEAULIEU}

\section{Structural Unemployment Theory and Measurement, by G. Peter Penz, Pro- gram Development Service, Depart- ment of Manpower and Immigration, Ottawa, 1969. Pp. ix +91 .}

Structural unemployment was a major topic of controversy during the sixties. More recently, the controversy seems to have been settled largely in favour of the aggregate demand school which holds the view that management of the economy should be based primarily, though not exclusively, on the traditional fiscal/monetary tools of stabilisation policy.

It appears, however, that structuralist sympathies have left deep roots in the Canada Department of Manpower and Immigration, which has recently sponsored the publication of a new book on the subject.

The book, based on the author's M.A. Thesis written at the University of British Columbia, is primarily a theoretical work. The longest chapter (Chapter II) is a survey of literature - a task which was adequately performed by others before, notably by J.W.L. Winder in The Canadian Labour Market edited by A. Kruger and N.M. Meltz, which book the Canada Department of Manpower and Immigration had also sponsored (in conjuction with the Centre for Industrial Relations at the University of Toronto).

Perhaps the most noteworthy aspect of this book is its attempt at utilizing job vacancy data for the measurement of Canadian structural unemployment (Chapters III and IV). Drawing upon some partial vacancy data from the old N.E.S. sources and the now defunct DBS Hirings and Separations survey, the author estimates that structural unemployment in Canada was nearly $4 \%$ in the 1960 's, up from $3 \%$ in the early 1950's (p. 87).

This might be an opportune moment to refer to the current state of Canadian job vacancy survey which is being jointly developed by the DBS and the Department of Manpower and Immigration. Once regular data are released, labour market analysis, both for theoretical and policy purposes, will be significantly stimulated. This will be particularly true if the vacancy data could be made available within a labour turnover context. To satisfy this last requirement, a new substitute will be needed for the old Hirings and Separation survey.

\section{Ozay MEHMET}

La médecine en mutation, par Dr Jacques Ménétrier, Collection MutationsOrientations, Casterman/poche, Paris, 1970, 159 pp.

L'auteur distingue la médecine orthodoxe de la médecine hétérodoxe.

La médecine orthodoxe, c'est aussi la médecine officielle, la médecine classique. L'auteur retrace les principaux jalons qui permettent de suivre l'évolution de cette voie dite «conforme». Il y eut d'abord l'évolution pasteurienne, caractérisée par la mise en évidence des agents microbiens, la certitude acquise d'une agression et le développement de l'immunologie. Ce sont les débuts de la médecine offensive. Cette ère se poursuit avec la découverte des antibiotiques et de la chimoithérapie. Toujours le pathologique est l'objet essentiel de la médecine, le fonctionnel n'occupe qu'une place de seconde importance. Cette voie se caractérise encore par la méconnaissance de l'immatériel et par le danger de confondre le malade et la maladie.

Par opposition à cette voie « conforme », il existe une deuxième voie dans 